\title{
An investigation on the application and challenges for wide area monitoring and control in smart grid
}

\author{
Marwan Ahmed Abdullah Sufyan, Mohd Zuhaib, Mohd Rihan \\ Department of Electrical Engineering, ZHCET College, Aligarh Muslim University (AMU), Aligarh, India
}

\begin{tabular}{l} 
Article Info \\
\hline Article history: \\
Received Oct 1, 2020 \\
Revised Dec 23, 2020 \\
Accepted Jan 27, 2021 \\
\hline
\end{tabular}

\section{Keywords:}

Global positioning system Phasor data concentrator Phasor measurement unit Supervisory control and data acquisition system Voltage stability monitoring Wide area monitoring and control

\begin{abstract}
The complexity and dynamics of the modern power system are continuously changing due to the penetration of a large number of renewable energy sources and changing load patterns. These growing complexities have caused numerous outages around the world, primarily due to the lack of situational awareness about the grid operating states. Rectification of this problem requires advanced sensing technology to accurately capture the dynamics of the system for better monitoring and control. Measurement of synchrophasors is a potential solution to improve situational awareness in the grid. The synchrophasors technology is now widely accepted throughout the world and has the potential to replace the existing SCADA system in monitoring and control of the power system. Their installation enables efficient resolution to substantially improve transmission system planning, maintenance, operation, and energy trading. This paper reviews the state of the art potential applications that the PMU based WAMC offers to the power system. It also includes technical perspectives, challenges, and future possibilities.
\end{abstract}

This is an open access article under the CC BY-SA license.

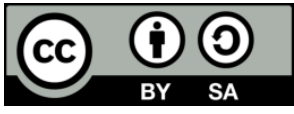

\section{Corresponding Author:}

Mohd Zuhaib

Department of Electrical Engineering

Aligarh Muslim University

Aligarh, 202002, UP, India

Email: mohd.zuhaib@zhcet.ac.in

\section{INTRODUCTION}

Two severe power outages influenced a large portion of India's northern and eastern parts on July 30-31, 2012. These outages influenced more than 40 corers people and are considered the most massive electricity blackouts in history regarding the number of individuals influenced [1]. About $32 \mathrm{GW}$ of the total load was taken offline [2]. Therefore, advanced power system monitoring and control techniques are required that enhances the situational awareness of the grid operator to ensure power system reliability against failure. Wide area monitoring and control (WAMC) is an advanced approach to forecast or detect the disastrous failure of a power system advancing from a minor fault. When a fault occurs, the control signals from the WAMC will be sent to devices to take corrective actions to mitigate the fault at the earliest. The signals that are sent from WAMC should be timely and efficient [3].

In a modern power system, WAMC has been used to serve different purposes-for example, security, stability, state estimation, and load scheduling [4]. The accessibility of synchronous data from synchronized PMU measurements provide a coherent picture of the entire region and allows the grid operator to analyze the system's dynamics before and after the disturbance [5]. One of the severe issues in the use of PMU is data handling. One PMU with 20 measurements at 60 to 120 samples per second generates around 1 GB or 2 GB of data per day. Therefore, it is quite challenging for the central PDC at the control center to sort and analyze 
data from multiple locations [6, 7]. Synchronized PMUs have tremendous scope in power system stability and improving its dynamics [8]. One of the main problems in using WAMC as an instrument for stability improvement is the delay in signal transmission. The dependability of the communication grid is another significant problem with WAMC based control [9]. WAMC also gives the advantage to analyze and characterize some dynamic qualities of the interconnected power system, such as transients, low-frequency oscillations(inter-area, local, or forced) [10]. Whenever a fault occurs in the system, the WAMC utilizes the online estimation information to recognize the bus closest to the fault, and consequently, the faulty line is disconnected. Synchronized PMUs are playing a significant role in research related to power system transient stability. The information extracted from PMU data can be utilized to differentiate between various faults occurring in the power system, determination of inter-area damping computations, just as state estimation for dynamic power system stability studies $[11,12]$. Hence, PMU based WAMC encourages to develop real-time applications that enhance the awareness of grid operators against grid operating states.

The paper is divided into the following sections. Section 2 discusses the various state of the art applications offered by synchrophasors technology in the smart grid. Section 3 provides an overview of different communication infrastructures for PMU data transfer. It also discusses limitations due to delays associated at various stages throughout the channel's length and their remedial measures. The conclusion is drawn in section 4.

\section{SYNCRHOPHASORS TECHNOLOGY APPLICATION}

Synchrophasors technology is a time-synchronized technology that measures both the phase angle and magnitude of voltage in a power system that is time-synchronized to capture the coherent picture of the entire grid. These are estimated by high-speed devices called PMUs [13]. The schematic diagram of the PMU based wide-area monitoring and control system is shown in Figure 1. It plays a vital role in safe and reliable gird operation. Whenever fault appears in the system, the wide-area security system uses the online measurement data to discover the faulty buses and, consequently, the faulted line to isolate it from the rest of the system. They play a significant role in modern power system studies; the crucial ones are discussed in the following subsections $[14,15]$.

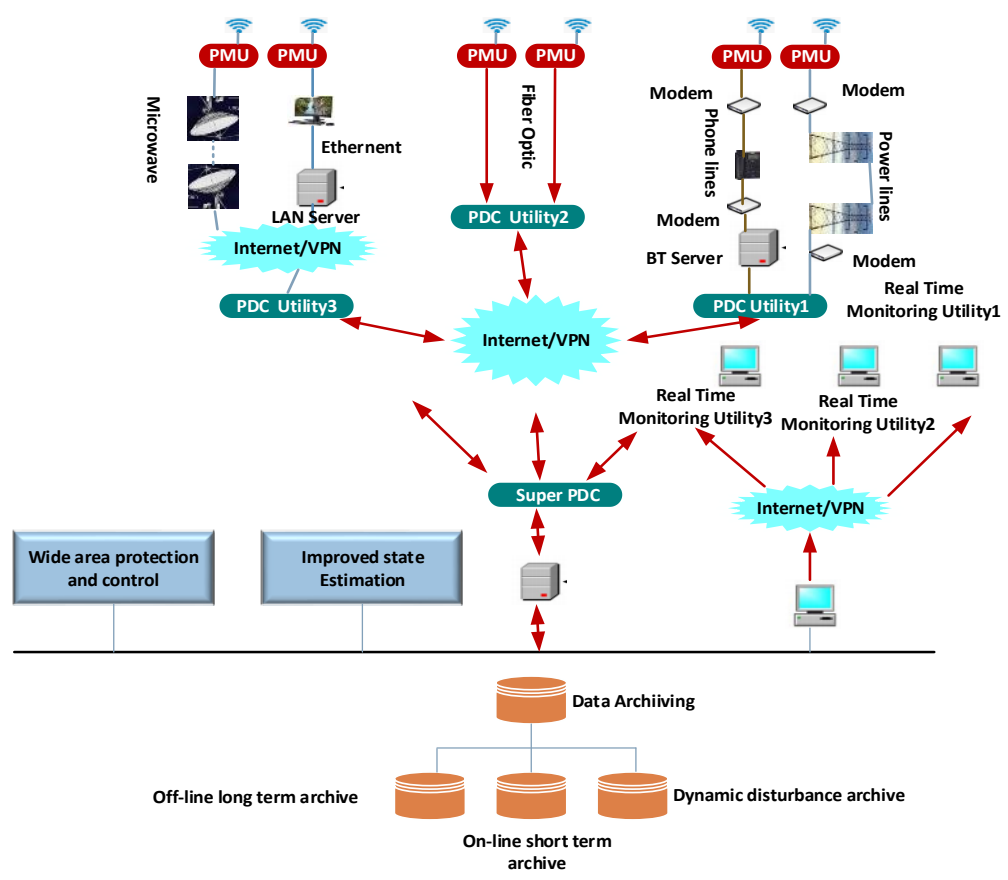

Figure 1. WAMC architecture [16]

\subsection{Voltage stability monitoring}

The purpose of voltage stability monitoring (VSM) is to estimate the degree of power system stability continuously. It is designed based on the selection of specific critical grid parameters and their stability index. This voltage stability index parameter includes [17-19]: 
- Voltage magnitude at critical locations

- Loading margin of the power system at the operating point

- Reactive power reserves

- Modal analysis parameters, e.g., damping ratio, dominant modes, and their participation factor in the abnormal system states.

Their functionality depends on comparing the computed stability index of the chosen critical parameters with their threshold value. The basic fundamental blocks of a typical VSM system are shown in Figure 2.

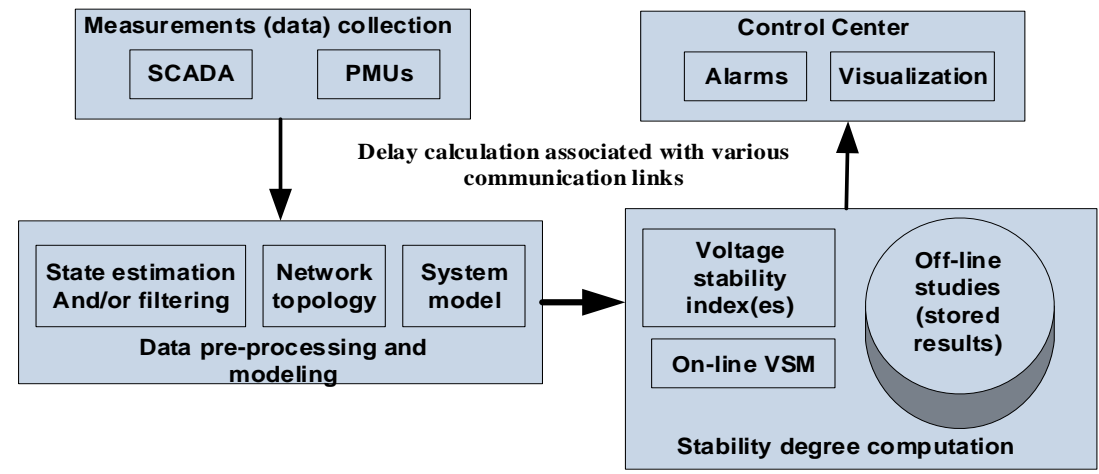

Figure 2. Essential components of a VSM [19]

The only solution to overcome the voltage stability problem is by providing reactive power support using the flexible AC transmission system (FACT) devices. These devices are classified as SVC, STATCOM, TCSC, UPFC, and can play a crucial role in overcoming voltage instability problems. The most efficient among them are SVC and STATCOM. They inject or absorb VARs through voltage source converters and provide instant support to voltage instability problems. These are also called dynamic VAR compensation devices. STATCOM provides faster response compared to SVC and is more resilient to frequent power system changes such as harmonic variations, and requires compact outdoor equipment compared to SVC. In high voltage condition, STATCOM operates in inductive mode and absorb reactive power while in low voltage conditions, it operates in capacitive mode and injects reactive power in the system [20].

\subsection{Oscillation monitoring system}

Based on WAMC, oscillation monitoring system (OMS) is an electrical supply system to monitor and examine system-wide dynamic phenomena continuously. Oscillations in power networks can be classified as electromechanical oscillations and forced oscillations [21]. Electromechanical oscillations occur in a power system fundamentally because of the large variation in generation and demand, faults, while forced oscillations are generally brought about by some external input driving the system rather than the abnormal state of the system itself. Ineffective damped oscillations can prompt abnormal system conditions, leading to system instabilities, eventually causing large power outages. For example, the July 31, 2012, power outage of the northern and eastern parts of India [12]. The goal of OSM is to discover poorly damped oscillations as they can affect system power flows and reduces power system reliability [22, 23] Studied how the OMS is extracting the data of electromechanical oscillations from PMU in real-time. PMU information is useful in recognizing the undamped electromechanical oscillations because of its high sampling rate. Khalilinia and Venkatasubramanian [24] discussed the denoising of the PMU data utilizing a wavelet shrinkage-based strategy. Hilbert's investigation is utilized to distinguish non-direct patterns in the denoised information. PMUs based low pass filter is also utilized to evacuate the anti-aliasing frequency segment.

Conventionally, damping of low-frequency oscillations is done with local control using generator speed deviation as a feedback signal. But it is insufficient to damp out inter-area oscillations due to inadequate information of inter-area oscillations present in the system. To overcome this problem, WAMC based wide area damping controller (WADC is quite useful due to its ability to accurately monitor system inter-area mode. The systematic diagram of such a system is shown in Figure 3. The PMUs in the WAMC acts as a sensor and estimate the modal analysis parameters such as dominant oscillation modes, mode shapes, and participation factor. Based on the identified system generator contributing to inter-area mode, control commands from WADC are sent to power system stabilizer to respective generators and FACT devices to damp out determined low-frequency oscillation modes [25]. 


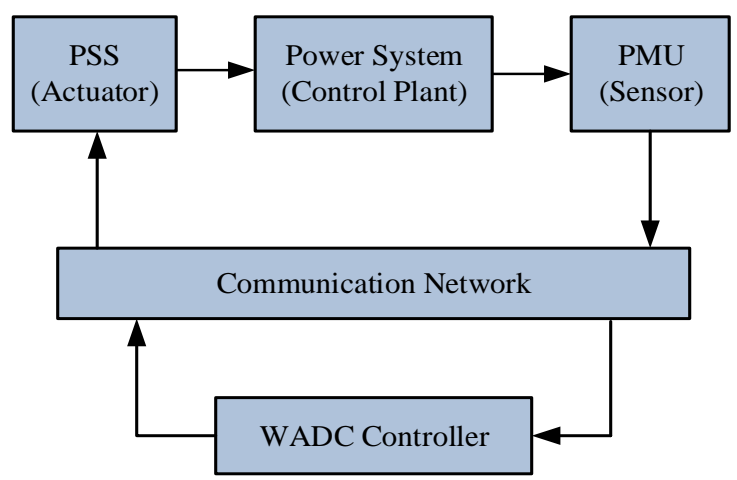

Figure 3. Structure of the WADC system [25]

\subsection{Fault detection and location}

The fault in a power system grows gradually. However, some phenomena, for example, transient instability, can evolve in a small time frame. Any adaptive fault detection algorithm for any protection/control system utilizes synchronized phasor estimates. On event occurrence with accessible measurements, any fault detection and isolation methodology should comprise of the following components [26]:

- Identification and forecasting: A quick recognizable detection of the specific event from power system parameters is desired to be measured to restore healthy power system states.

- Classification: Disturbance classification depends on the constraints that are violated, the risk involved, the time span of the phenomena, and utility control strategy.

- Control action: These are mainly operated based on the level of priority supplies during an emergency and maintain the grid's stable operation. They improve system reliability and ensure the social and economic aspects.

- Corrections: The system can be in an improved state after monitoring and correction measures have been applied. e.g., load shedding can be estimated to prevent the system from rapid frequency decline.

- Time scale: For any past events, accessible time is the fundamental factor in choosing correction activities. It includes selective implementation of remedial measures.

The fault location methods widely used in the power system can be broadly classified as impedancebased methods, traveling wave-based methods, and artificial intelligence-based methods. The impedance method uses voltage phasors to predict the location of a fault. The most important advantage of this method is that only one PMU is sufficient to locate the fault. However, they require prior fault assumptions or sections, which are quite complex to implement [27, 28]. Since fault results in the inception of transient, the traveling wave method utilizes its high frequency to estimate its arrival time from the fault point. To achieve this, wave velocity needs to be estimated from the fault point to the measuring device. These methods are independent of the type of fault that occurred in a power system. However, accurately estimating the wave voltage is a challenging task [29]. Artificial intelligence-based methods utilize voltage pattern recognition and machine learning techniques. The determination of fault location is independent of system parameters. It relies on data containing certain features of fault. This method is widely accepted due to its high accuracy and independence of system parameters [30].

\section{COMMUNICATION TECHNOLOGIES}

Communication links utilized by WAMC include wireless (microwave, cellular, satellite) and wired (fiber-optics, power lines, telephone lines) options. Delays related to communication technology are the crucial parameters for the selection of a particular communication medium and should be incorporated in every power system design to ensure proper control procedures to maintain the stability of the power system. Various wires and wireless communication technologies are given in Figure 4, and the delay associated with each technology is tabulated in Table 1. The delays are a significant perspective and ought to be consolidated into any power system design, or analysis and presence of sufficient delay could destroy any control strategies received to stabilize the power network [31,32]. The evolution of communication technology in the power system is given in Figure 5. 


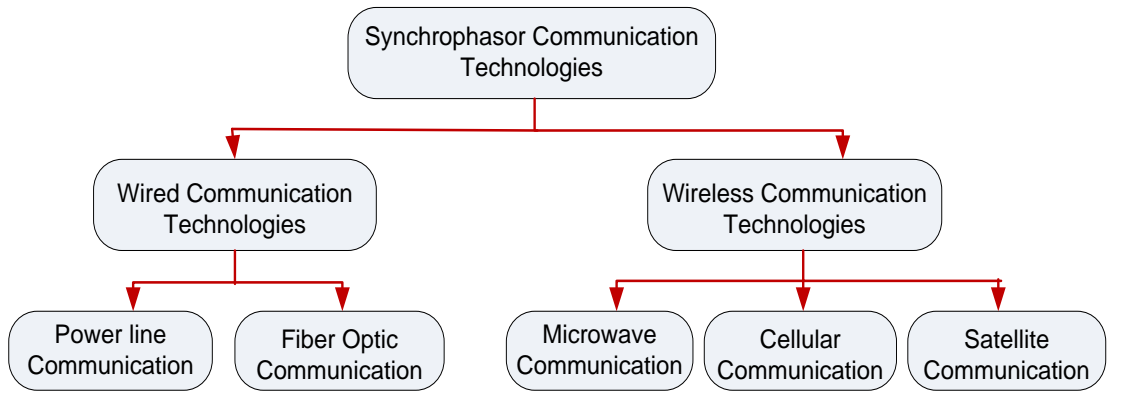

Figure 4. The various synchrophasors communication technologies [31]

Table 1. Delay associated with various communication links [33]

\begin{tabular}{lc}
\hline Communication link & $\begin{array}{c}\text { Associated delay one } \\
\text { way }(\mathrm{ms})\end{array}$ \\
\hline Fiber-optic cables & $\approx 100-150$ \\
Digital microwave & $\approx 100-150$ \\
links & $\approx 150-350$ \\
Power line carrier & \\
(PLC) & $\approx 200-300$ \\
Telephone lines & $\approx 500-700$ \\
Satellite link &
\end{tabular}

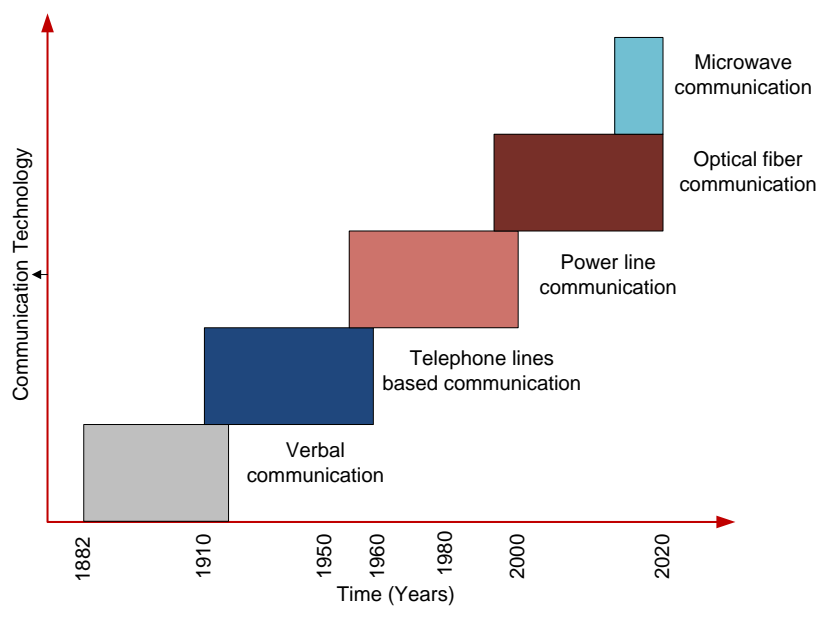

Figure 5. Evolution of communication technology in power system [31]

A WAMC system's performance largely relies upon the time delay of transmitting information through the communication grid. Figure 6 shows the effect of these delays in the fault clearance process at $400 \mathrm{KV}$ Kanpur bus of the Indian power grid. As can be observed from Figure 6, the delay in fault clearance is around $520 \mathrm{~ms}$, which is much higher as prescribed by the Indian electricity grid code (IEGC). The time delay should be minimized by using efficient communication infrastructure to avoid failures [34]. The selection of communication media and the spaces of communication have an impact on the spread of delay. A representation of these delays in WAMC is shown in Figure 7. Among all delays, propagation and processing delays have a significant effect on the performance of the system.

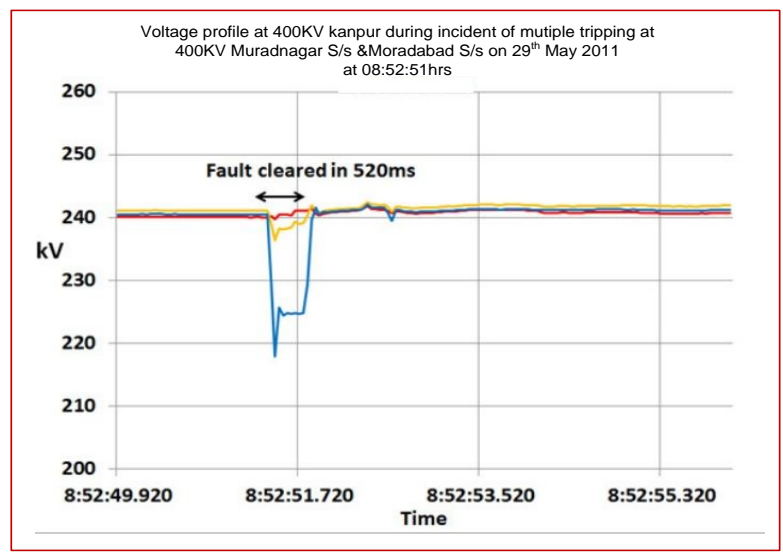

Figure 6. Detection of delay fault clearance [33] 


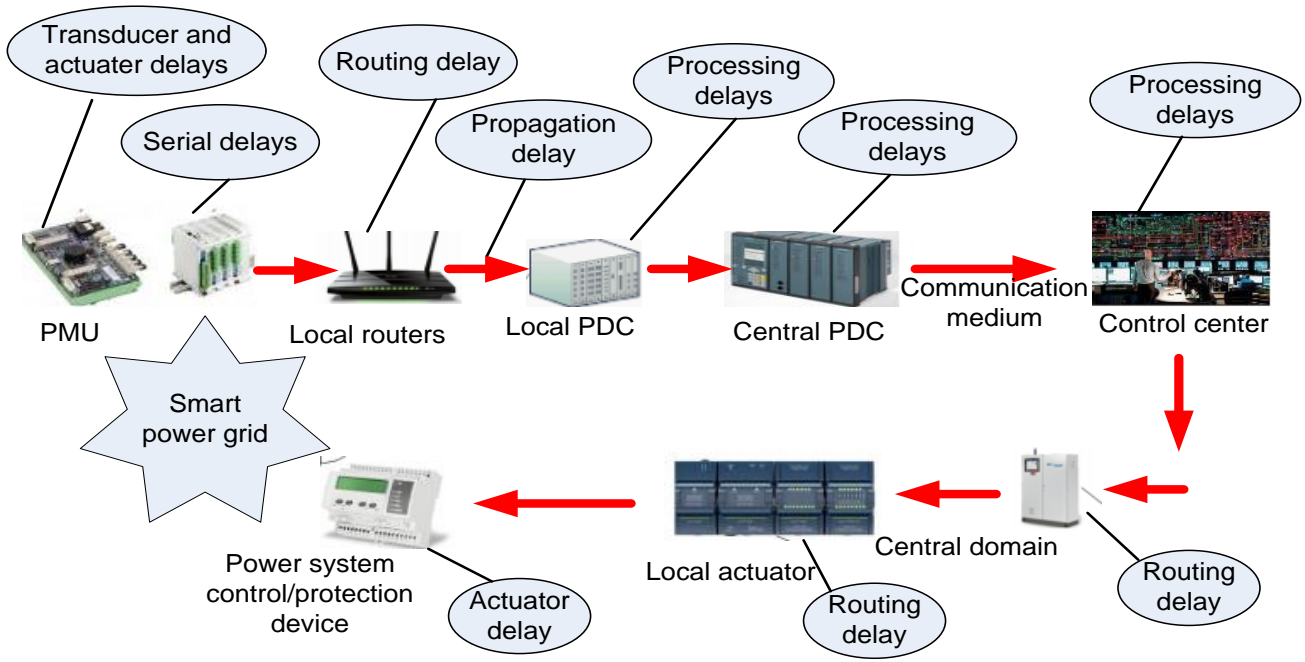

Figure 7. Classification of different delays connected with PMU based WAMC [35]

Various research works have been carried out in the literature to determine their root causes and suggest remedial measures. Appasani and Mohanta [36] proposed the RTD model of the WAMC framework considering various parameters resulting in communication delays and data loss throughout the channel. It considers the time delay of data sampling of PMUs, information spread in the communication system, and information received at the control centre. Singh et al. [37] discussed an algorithm to estimate time delay and packet data loss using a Markov model to achieve better control. Zhu et al. [38] studied the design of the supporting information and communication technology (ICT) framework for WAMC applications. It suggested a technique to achieve better control with inefficient available measurements due to time delays, packet loss, or bandwidth constraints. Zhu et al. [39] proposed a self-adaptive PDC that compensates for time delays in the communication system. Xiaorong et al. [40] Explains the communication delays throughout the length of the communication system, i.e., from PMUs to synchrophasors arranged by PDC. The outcomes recommend that a bi-modular transmission containing two standard transmissions shows the best fit to avoid the time delay problem.

\section{CONCLUSION AND FUTURE WORK}

This paper provides a comprehensive assessment of WAMC that helps in ensuring a secure and reliable grid operation. Several potential applications such as voltage stability monitoring, oscillation detection, andfault detection, that WAMC offers to the grid have been discussed. However, there are certain potential challenges in its large-scale implementation. It includes integrating PMU data with data provided by SCADA, the requirement of advanced communication infrastructure to handle massive data set generated by PMUs, and delays associated with the communication channel.

Apart from various applications offered by synchrophasors technology at the transmission side, its diffusion in the distribution system is facing potential challenges due to variable grid characteristics and financial limitations due to a large number of nodes. To overcome such challenges and to keep in mind the increasing availability of power electronic converters due to the increasing trend of renewable energy sources integration, the implementation of $\mu \mathrm{PMU}$ capabilities in the grid connected converters can be a potential research area. This reduces the high cost on large-scale implementation of PMUs across the distribution system. The aim can be increasing the functionality of power converters utilizing PMU data to provide additional auxiliary services in addition to the primary task of active power injection into the grid, islanding detections, and maximum power point tracking, that can make the distribution system more robust and resilient.

\section{REFERENCES}

[1] Helen Pidd, "India blackouts leave 700 million without power," The Guardian, 2012. [Online]. Available: https://www.theguardian.com/world/2012/jul/31/india-blackout-electricity-power-cuts.

[2] India's mass power failure worst ever in world history, Outlook: Press Trust of India, 2012. [Online]. Available: https://www.outlookindia.com/newswire/story/indias-mass-power-failure-worst-ever-in-world-history/770631. 
[3] M. Rihan, M. Ahmad and M. S. Beg, "Phasor measurement units in the Indian smart grid," ISGT2011-India, pp. 261-267, 2011.

[4] A. Obushevs and A. Mutule, "Application of synchrophasor measurements for improving situational awareness of the power system," Latvian Journal of Physics and Technical Sciences, vol. 55, no. 2, pp. 3-10, 2018.

[5] M. Zuhaib and M. Rihan, "PMU installation in power grid for enhanced situational awareness: Challenges, issues and application," 2017 4th IEEE Uttar Pradesh Section International Conference on Electrical, Computer and Electronics (UPCON), pp. 654-659, 2017.

[6] A. von Meier, E. Stewart, A. McEachern, M. Andersen, and L. Mehrmanesh, "Precision micro-synchrophasors for distribution systems: A summary of applications," IEEE Transactions on Smart Grid, vol. 8, no. 6, pp. 2926-2936, Nov. 2017.

[7] Mohd Zuhaib, Abul Khair, and Mohd Rihan, "An objective analysis of micro-synchrophaosrs technology for monitoring and control of active distribution network," Journal of the Institution of Engineers (India): Electrical Engineering Division, vol. 3, no. 1, pp. 27-36, 2019.

[8] M. A. Abdullah Sufyan, M. Zuhaib, M. Sefid, and M. Rihan, "Analysis of effectiveness of PMU based wide area monitoring system in Indian power grid," 2018 5th IEEE Uttar Pradesh Section International Conference on Electrical, Electronics and Computer Engineering (UPCON), pp. 1-6, 2018.

[9] Abul Khair, Mohd Zuhaib, and Mohd Rihan, "Effective utilization of limited channel PMUs for islanding detection in a solar PV integrated distribution system," Journal of The Institution of Engineers (India): Series B, pp. 1-12, 2020.

[10] X. Wang and K. Turitsyn, "Data-driven diagnostics of mechanism and source of sustained oscillations," IEEE Transactions on Power Systems, vol. 31, no. 5, pp. 4036-4046, Sept. 2016.

[11] M. Rhodes, R. Quint, and A. Silverstein, "Integrating synchrophasor technology into power system protection applications," 2019 International Conference on Smart Grid Synchronized Measurements and Analytics (SGSMA), pp. 1-8, 2019.

[12] M. U. Usman and M. O. Faruque, “Applications of synchrophasor technologies in power systems,” Journal of Modern Power Systems and Clean Energy, vol. 7, no. 2, pp. 211-226, March 2019.

[13] A. Khair, M. A. A. Sufyan, M. Zuhaib, and M. Rihan, "PMU assisted state estimation in distribution system with PV penetration," 2019 International Conference on Electrical, Electronics and Computer Engineering (UPCON), pp. 1-5, 2019.

[14] Abul Khair, Mohd Rihan, and Mohd Zuhaib, "Implementation of controlled islanding scheme for self-healing smart grid," International Journal of Engineering \& Technology, vol. 7, pp. 945-950, 2018.

[15] S. Hampannavar, C. B. Teja, M. Swapna, and U. Kumar R. Y., "Performance improvement of M-class phasor measurement unit (PMU) using Hamming and Blackman windows," 2020 IEEE International Conference on Power Electronics, Smart Grid and Renewable Energy (PESGRE2020), pp. 1-5, 2020.

[16] V. Terzija, G. Valverde, D. Cai, P. Regulski, V. Madani, J. Fitch, S. Skok, M. M. Begovic, and A. Phadke, "Widearea monitoring, protection, and control of future electric power networks," Proceedings of the IEEE, vol. 99, no. 1, pp. 80-93, Jan. 2011.

[17] J. Jung, H. Cho, B. Park, S. Nam, K. Hur, and B. Lee, "Enhancement of linearity and constancy of PMU-based voltage stability index: Application to a Korean wide-area monitoring system," IET Generation, Transmission \& Distribution, vol. 14, no. 17, pp. 3357-3364, 2020, doi: 10.1049/iet-gtd.2019.0773.

[18] J. D. Pinzón and D. G. Colomé, "Data analytics of PMU measurement features for real-time short-term voltage stability prediction," 2019 FISE-IEEE/CIGRE Conference-Living the energy Transition (FISE/CIGRE), pp. 1-6, 2019, doi: 10.1109/FISECIGRE48012.2019.8985004.

[19] M. K. H. Pulok and M. O. Faruque, "Utilization of PMU data to evaluate the effectiveness of voltage stability boundary and indices," 2015 North American Power Symposium (NAPS), pp. 1-6, 2015, doi: 10.1109/NAPS.2015.7335111.

[20] Y. Lee and H. Song, "A reactive power compensation strategy for voltage stability challenges in the Korean power system with dynamic loads," Sustainability, vol. 11, no. 2, pp. 326-1-19, 2019, doi: 10.3390/su11020326.

[21] J. Follum and J. W. Pierre, "Detection of periodic forced oscillations in power systems," IEEE Transactions on Power Systems, vol. 31, no. 3, pp. 2423-2433, May 2016.

[22] M. Zuhaib, M. Rihan, and Mohd T. Saeed, "A novel method for locating the source of sustained oscillation in power system using synchrophasors data," Protection and Control of Modern Power Systems, vol. 5, no. 1, pp. 112,2020

[23] M. K. Arpanahi, M. Kordi, R. Torkzadeh, H. H. Alhelou, and P. Siano, "An augmented prony method for power system oscillation analysis using synchrophasor data," Energies, vol. 12, no. 7, pp. 1267-1-17, 2019.

[24] H. Khalilinia and V. Venkatasubramanian, "Modal analysis of ambient PMU measurements using orthogonal wavelet bases," IEEE Transactions on Smart Grid, vol. 6, no. 6, pp. 2954-2963, Nov. 2015

[25] X. Zhang, C. Lu, S. Liu, and X. Wang, "A review on wide-area damping control to restrain inter-area low frequency oscillation for large-scale power systems with increasing renewable generation," Renewable and Sustainable Energy Reviews, vol. 57, pp. 45-58, 2016.

[26] A. E. L. Rivas and T. Abrão, "Faults in smart grid systems: Monitoring, detection and classification," Electric Power Systems Research, vol. 189, p. 106602, 2020.

[27] M. M. Devi, M. Geethanjali, and A. R. Devi, "fault localization for transmission lines with optimal phasor measurement units," Computers \& Electrical Engineering, vol. 70, pp. 163-178, 2018. 
[28] S. Affijulla and P. Tripathy, "A robust fault detection and discrimination technique for transmission lines," IEEE Transactions on Smart Grid, vol. 9, no. 6, pp. 6348-6358, Nov. 2018.

[29] M. Daryalal and M. Sarlak, "Fast fault detection scheme for series-compensated lines during power swing," International Journal of Electrical Power \& Energy Systems, vol. 92, pp. 230-244, 2017.

[30] S. Das, S. P. Singh, and B. K. Panigrahi, "Transmission line fault detection and location using wide area measurements,” Electric Power Systems Research, vol. 151, pp. 96-105, 2017.

[31] B. Appasani and D. K. Mohanta, "A review on synchrophasor communication system: Communication technologies, standards and applications," Protection and Control of Modern Power Systems, vol. 3, no. 1, pp. 37, 2018.

[32] S. M. Blair, M. H. Syed, A. J. Roscoe, G. M. Burt, and J. Braun, "Measurement and analysis of PMU reporting latency for smart grid protection and control applications," IEEE Access, vol. 7, pp. 48689-48698, 2019, doi: 10.1109/ACCESS.2019.2903929.

[33] B. Naduvathuparambil, M. C. Valenti, and A. Feliachi, "communication delays in wide area measurement systems," Proceedings of the Thirty-Fourth Southeastern Symposium on System Theory (Cat. No.02EX540), pp. 118-122, 2002.

[34] "Synchrophasor Initiative in India," Power System Operation Corporation Limited, December-2013.

[35] A. S. Musleh, S. M. Muyeen, A. Al-Durra, I. Kamwa, M. A. S. Masoum, and S. Islam, "Time-delay analysis of wide-area voltage control considering smart grid contingences in a real-time environment," IEEE Transactions on Industrial Informatics, vol. 14, no. 3, pp. 1242-1252, March 2018.

[36] B. Appasani and D. K. Mohanta, "Co-optimal placement of PMUs and their communication infrastructure for minimization of propagation delay in the WAMS," IEEE Transactions on Industrial Informatics, vol. 14, no. 5, pp. 2120-2132, May 2018.

[37] V. P. Singh, N. Kishor, and P. Samuel, "Communication time delay estimationfor load frequency control in twoarea power system," Ad Hoc Networks, vol. 41, pp. 69-85, 2016.

[38] K. Zhu, M. Chenine, L. Nordström, S. Holmström, and G. Ericsson, "An empirical study of synchrophasor communication delay in a utility TCP/IP network," International Journal of Emerging Electric Power Systems, vol. 14, no. 4, pp. 341-350, 2013.

[39] K. Zhu, S. Rahimi, L. Nordström, and B. Zhang, "Design phasor data concentrator as adaptive delay buffer for wide-area damping control," Electric Power Systems Research, vol. 127, pp. 22-31, 2015.

[40] C. Xiaorong, W. Ying, and N. Yangdan, "the study on the communication network of wide area measurement system in electricity grid," Physics Procedia, vol. 25, pp. 1708-1714, 2012. 